\title{
Robotic Assisted Laparoscopic Partial Cystectomy and Urachal Resection for Urachal Adenocarcinoma
}

\author{
Philippe E. Spiess, Jose J. Correa \\ Division of Urology, H. Lee Moffitt Cancer Center, Tampa, Florida, USA
}

\begin{abstract}
Introduction and Objective: Standard treatment for urachal adenocarcinomas is open partial cystectomy and urachal resection; however, minimally invasive surgical approaches including laparoscopic and recently described robotic assisted laparoscopic partial cystectomy and urachal resection is feasible with potential less morbidity. A case of robotic assisted partial cystectomy and urachal resection for urachal adenocarcinoma is presented. Few articles in the literature have being published describing this technique and to the best of our knowledge, this is the largest and potentially most complex case approached in such a manner.

Methods: A 55 years old African American male presented with hematuria and mucosuria, cystoscopy demonstrated a tumor involving the dome of the bladder. Transurethral biopsy confirmed a urachal adenocarcinoma. Further studies revealed a negative metastatic evaluation. Preoperative abdominal/pelvic CT imaging revealed an enhancing mass extending from the inferior level of the umbilicus to the dome of the bladder. A total of 6 laparoscopic ports were used. The robotic assisted laparoscopic dissection was started at the level of the umbilicus, dissecting lateral to the right and left medial umbilical ligaments up until the dome of the bladder. A simultaneous cystoscopy with transillumination to define the bladder boundaries of this mass, with robotic assisted laparoscopic opening of the bladder, with the entire mass (including bladder component) excised and sent for frozen pathology for margin evaluation. After specimen extraction, the bladder was closed in two layers. Total surgery time was 300 minutes and intra-operative blood loss was $150 \mathrm{cc}$.

Results: Final pathology reported a pT2N0Mx adenocarcinoma with negative margins and negative pelvic lymph nodes. Patient was started on clear liquids on postoperative day 2 and on regular diet on postoperative day 3 . He was discharged on postoperative day 4 . A cystogram perfomed on postoperative day 7 revealed a good bladder capacity $(350 \mathrm{cc})$ and no leakage was identified.

Conclusions: Robotic assisted partial cystectomy and urachal resection for urachal adenocarcinoma of the bladder is feasible even in challenging cases. This surgical approach is less morbid in terms of postoperative pain and cosmesis when compared to the open standard approach. The postoperative recovery is faster; however, application of oncological principles and comfort with laparoscopic and robotic surgery is needed prior to attempting such challenging cases.
\end{abstract}

Int Braz, J Urol. 2009; 35 (Video \#1): 609

Available at: www.brazjurol.com.br/videos/september_october_2009/Spiess_609

Correspondence address:

Accepted:

Dr. Philippe E. Spiess

August 8, 2009

Division of Urology

H. Lee Moffitt Cancer Center

Tampa, Florida, USA

E-mail: Philippe.Spiess@moffitt.org 